\title{
Moringa oleifera Seeds Attenuate Vascular Oxidative and Nitrosative Stresses in Spontaneously Hypertensive Rats
}

\author{
Joseph Iharinjaka Randriamboavonjy, ${ }^{1,2}$ Marc Rio, ${ }^{1}$ Pierre Pacaud, ${ }^{1}$ \\ Gervaise Loirand, ${ }^{1,2}$ and Angela Tesse ${ }^{1}$ \\ ${ }^{1}$ INSERM, CNRS, Université de Nantes, l'institut du thorax, 44000 Nantes, France \\ ${ }^{2} \mathrm{CHU}$ de Nantes, 44000 Nantes, France \\ Correspondence should be addressed to Angela Tesse; angela.tesse@univ-nantes.fr
}

Received 20 March 2017; Accepted 3 May 2017; Published 20 June 2017

Academic Editor: Vincenzo Lionetti

Copyright (c) 2017 Joseph Iharinjaka Randriamboavonjy et al. This is an open access article distributed under the Creative Commons Attribution License, which permits unrestricted use, distribution, and reproduction in any medium, provided the original work is properly cited.

\begin{abstract}
Moringa oleifera (MOI) is a tree currently used in traditional medicine in tropical Africa, America, and Asia for therapeutic applications in several disorders including arterial hypertension. We previously described a cardiac protective role of a treatment with MOI seeds in spontaneously hypertensive rats (SHR). Here, we investigated the effects of this treatment on oxidative and nitrosative vascular stresses in SHR, with normotensive Wistar Kyoto rats used as controls. Oxidative and nitrosative stresses detected in SHR aortas using the fluorescent dye dihydroethidine and protein nitrotyrosine staining were reduced in MOItreated SHR aortas. This was associated with a decrease of free 8 -isoprostane circulating level, vascular p $22^{\text {phox }}$ and $\mathrm{p} 47^{\text {phox }}$ expressions, and SOD2 upregulation. Moreover, circulating nitrites and C-reactive protein, increased in SHR, were both reduced in SHR receiving MOI. This was associated to decrease iNOS and NF- $\kappa$ B protein expressions after MOI treatment. In functional studies, the endothelium-dependent carbachol-induced relaxation was improved in MOI-treated SHR resistance arteries. Oral administration of MOI seeds demonstrates vascular antioxidant, anti-inflammatory, and endothelial protective effects in SHR. Our data support the use of MOI seeds in diet against cardiovascular disorders associated with oxidative stress and inflammation such as hypertension, scientifically validating the use of these seeds in Malagasy traditional medicine.
\end{abstract}

\section{Introduction}

Hypertension is defined as a chronic elevated systolic and/or diastolic blood pressure [1]. High blood pressure is associated with an increased risk of cardiovascular morbidity and mortality and is even identified as the leading cause of avoidable mortality and morbidity worldwide $[2,3]$. Until recently, hypertension was mainly associated with developed countries. However, the condition is constantly growing in low- and middle-income countries. Currently, the global prevalence of high blood pressure in adults is around $22 \%$ but reaches 30\% in Africa where it is the main risk factor of premature death from stroke, cardiac, and renal failure [4]. The health benefits of lowering blood pressure, in particular the resulting reduction in cardiovascular morbidity and mortality, have been indisputably proven [5]. Countries of the African region are thus deploying effective programs and strategies, including traditional medicines, to promote detection, prevention, and control of high blood pressure.

In African countries, in particular in Madagascar, the traditional medicine is a practice based on empiric knowledge of the therapeutic use of plants transmitted from generation to generation. Malagasy medicinal plants are still often used as a first easily affordable resort to treat common disorders including hypertension $[6,7]$. For some of these plants, experimental evidence has proven real beneficial effects in the heart, vessels, and hemodynamic parameters. Cedrelopsis grevei (Meliaceae) has been shown to have antihypertensive properties via the improvement of endothelial function [8], and, most recently, Mimosa pigra (Fabaceae) has been 
reported to have protective vascular effect via its antioxidant properties in hypoxic rats developing pulmonary arterial hypertension [9].

Moringa oleifera (MOI) (Moringaceae) is a tree currently used by traditional medicine in tropical Africa, America, and Asia to treat systemic arterial hypertension [10].

The therapeutic but empirical use of medicinal plants in Madagascar is generally not supported by rational clinical or experimental data. Malagasy population employs MOI seeds against hypertension empirically, and in our previous study, we provided evidence of the cardiac protective role of a treatment with MOI seeds in spontaneously hypertensive rats (SHR) [11]. A physicochemical study suggested antioxidant properties of MOI seed oil [12]. MOI seed oil showed a concentration-dependent free radical scavenging activity probably due to the presence of molecules known to have antioxidant activities such as phenols and in particular flavonoids [12].

The aim of the present study was to assess the in vivo pharmacological efficacy of a diet containing MOI seeds to reduce oxidative and nitrosative stresses and vascular inflammation in SHR. Our results revealed antioxidant and antiinflammatory effects supporting the use of MOI seeds in diet to improve cardiovascular disorders associated with vascular oxidative stress and inflammation such as hypertension.

\section{Materials and Methods}

2.1. Animals. Sixteen-week-old male Wistar Kyoto rats (WKY) and SHR were used. Rats have been fed with either normal food (WKY and SHR groups) or food containing MOI seed powder (750 mg/day/rat; SHR MOI group) for 20 weeks. At 36 weeks of age, rats of the three groups were anesthetized by isoflurane inhalation to harvest blood samples for circulating free 8-isoprostane, nitrite, and C-reactive protein (CRP) measurements. Rats were then sacrificed; the thoracic aorta and mesenteric arteries were then collected for Western blot, immunohistological, and vascular reactivity analyses. All experiments were conducted in agreement with international guidelines for care and use of laboratory animals and approved by our Ethical Committee (authorisation number 00909.01).

2.2. Staining and Confocal Microscopy Imaging. Frozen sections of aorta ( $7 \mu \mathrm{m}$ thick) on glass slides were used to the in situ detection of superoxide anion $\left(\mathrm{O}_{2}^{-}\right)$with the oxidative fluorescent dye dihydroethidine (DHE, Sigma-Aldrich) as previously described by Miller et al. [13]. DHE oxidizes to $\mathrm{EtBr}$ in the presence of $\mathrm{O}_{2}^{-}$and shows a red fluorescence.

In another set of experiments, the vessel frozen sections were fixed with cold 100\% methanol and incubated ( $2 \mathrm{~h}$ at room temperature) in blocking buffer (5\% nonfat dry milk in PBS). Tissue sections were then incubated overnight $\left(4^{\circ} \mathrm{C}\right)$ with either a mouse monoclonal antibody antinitrotyrosine (1:100, Upstate Biothechnology), a monoclonal murine anti-iNOS ( $1: 50, \mathrm{BD}$ Transduction Laboratories), and a polyclonal NF- $\kappa$ B p65 antibody $(1: 100$, Cell Signaling), for protein nitrotyrosine, iNOS, or NF- $\kappa$ B p 65 immunostaining, respectively. Three washes were followed by incubation ( $1 \mathrm{~h}$, at room temperature, in the dark) with Alexa fluor-647 anti-mouse or anti-rabbit secondary antibody (1:500, Molecular Probes). A Nikon A1-RS inverted laser scanning confocal microscope was used for the optical sectioning of the tissue. Digital image recording was performed using the NIS element software. Images were analyzed and processed by Fiji software.

2.3. ELISA Measurements. Plasma measurements of free 8isoprostane and CRP were performed using ELISA assay kits from Cayman Chemical and Millipore, respectively.

2.4. NO Measurements. Circulating NO was determined from nitrite concentration $\left(\mathrm{NO}_{2}^{-}\right)$by a colorimetric assay according to the Griess reaction method (Griess Reagent Kit, Molecular Probes; Invitrogen).

2.5. Western Blot Analysis. Aortas were homogenized and lysed. Proteins (50-75 $\mu \mathrm{g}$ ) were separated on 12 or $14 \%$ SDS-PAGE electrophoresis gel, transferred to nitrocellulose membrane then probed with antibodies to $\mathrm{p} 22^{\text {phox }}, \mathrm{p} 47^{\text {phox }}$, gp91 ${ }^{\text {phox }}$, p67 ${ }^{\text {phox }}$, SOD1, or SOD2 (Santa Cruz Biotechnology, INC). A monoclonal anti- $\alpha$-tubulin antibody $(1: 5000$, Sigma-Aldrich) was used to check protein gel loading and to normalize protein expression. Immunoreactive bands were revealed with a secondary peroxidase-conjugated antimouse or anti-rabbit IgG (1:5000, Beckman Coulter), detected by enhanced chemiluminescence system (ECL Plus, Amersham Biosciences) and quantified by densitometry.

2.6. Arterial Reactivity. Aortas and first branches of superior mesenteric arteries were collected in physiological saline solution (in mM: $130 \mathrm{NaCl}, 5.6 \mathrm{KCl}, 1 \mathrm{MgCl}_{2}, 2 \mathrm{CaCl}_{2}, 11$ glucose, 10 Tris, pH 7.4 with $\mathrm{HCl}$ ), cleaned, and cut in $2 \mathrm{~mm}$ long rings. Arterial rings were then mounted on multichannel isometric myograph, bathed in Krebs-Henseleit solution at $37^{\circ} \mathrm{C}$, bubbled with $95 \% \mathrm{O}_{2}-5 \% \mathrm{CO}_{2}$, and connected to a force transducer (Pioden controls Ltd, Canterbury, UK for aortic rings; Danish Myo Technology; Aarhus, Danemark, for mesenteric artery rings). After equilibration, the contractile response to $\mathrm{KCl} 60 \mathrm{mM}$ was measured. Endothelial function was assessed by measuring the relaxing response to cumulative doses of carbachol (CCh, $1 \mathrm{nM}$ $-10 \mu \mathrm{M})$ of rings precontracted by phenylephrine (PhE, $1 \mu \mathrm{M})$. Digital data were recorded by a MacLab/4e recorder and analyzed using a LabChart v7 software (AD Instruments, Paris, France).

2.7. Data Analysis. A one-way ANOVA with subsequent Bonferroni post hoc test or the ANOVA on ranks were performed for all the experiments except for them of vascular reactivity for which we used a two-way analysis of variance for repeated measures with subsequent Bonferroni post hoc test. All statistical analyses were realized with Statview software (SAS Institute, Cary, NC, USA). All values are presented as mean \pm standard deviation for $n$ experiments, $n$ representing the number of rat samples. ${ }^{*} p<0.05$ was considered to be statistically significant. 

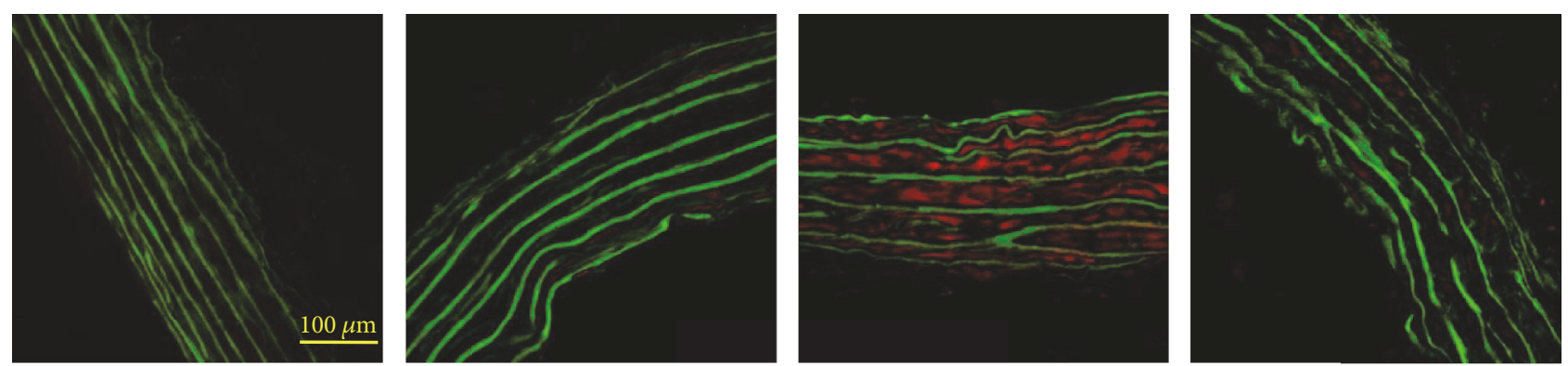

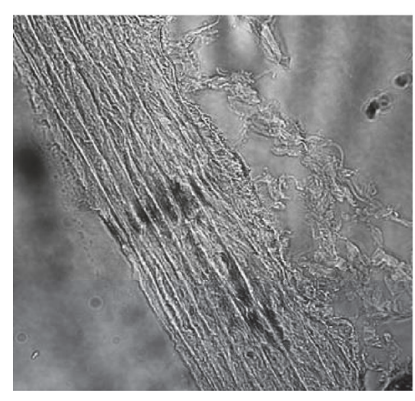

$\mathrm{C}(-)$

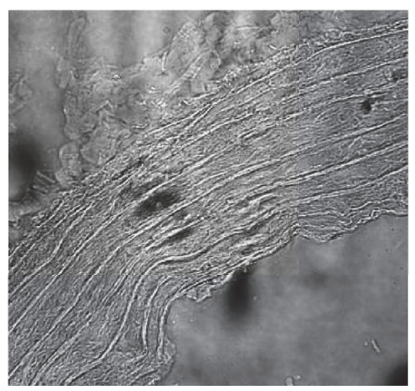

WKY

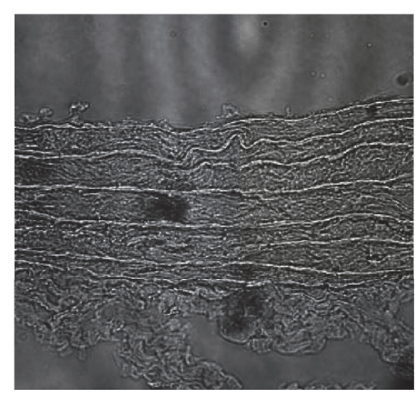

SHR

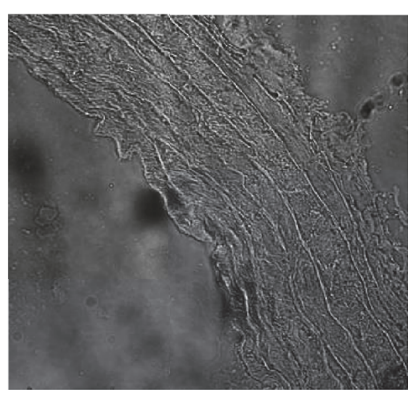

SHR MOI

(a)

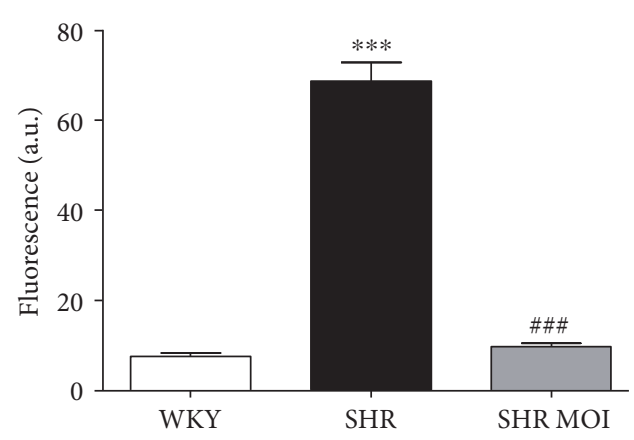

(b)

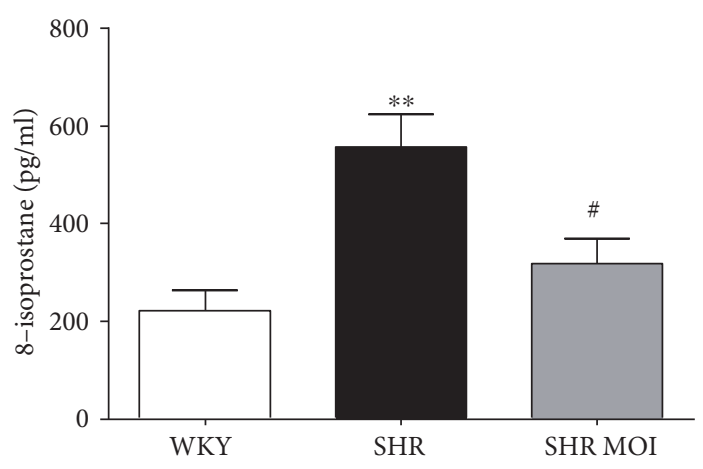

(c)

FIgURE 1: (a) Oxidative stress (red staining by DHE of $\mathrm{O}_{2}^{-}$in rat aortas from Wistar Kyoto (WKY), SHR untreated (SHR), and SHR treated with MOI (SHR MOI)). Green fluorescence corresponds to autofluorescence of elastin. Below phase-contrast images. Negative control C(-) without DHE. (b) Fluorescence is expressed in arbitrary units (a. u.), WKY (white plot), SHR (black plot), and SHR MOI (grey plot) ( $n=5$ for each group, ${ }^{* * *} p<0.001$ SHR versus WKY and ${ }^{\# \# \#} p<0.001$ SHR MOI versus SHR). (c) Circulating levels of free 8-isoprostane expressed in $\mathrm{pg} / \mathrm{ml}$ of plasma ( $n=6-7$ for each group, ${ }^{* *} p<0.01$ SHR versus WKY and ${ }^{*} p<0.05$ SHR MOI versus SHR).

\section{Results}

3.1. MOI Reduces Oxidative Stress in SHR Aortas. To assess the effect of MOI treatment on oxidative stress characterizing hypertensive rats, we directly assessed the in situ production and the topographical distribution of $\mathrm{O}_{2}^{-}$in aortic sections from WKY, SHR, and SHR MOI. As expected, compared to control WKY aortas, SHR aortas displayed a marked increase in EtBr fluorescence, reflecting elevated oxidative stress in the vascular wall (Figures $1(\mathrm{a})$ and $1(\mathrm{~b})$ ). In contrast, the $\mathrm{O}_{2}^{-}$ staining in aortic sections from SHR MOI was comparable to that from WKY rats (Figures 1(a) and 1(b)).

The antioxidant effect of MOI was further addressed by the measurement of free circulating 8-isoprostane, a plasmatic oxidative stress marker. In SHR, the plasmatic level of 8 -isoprostane was significantly increased compared to
WKY (Figure 1(c)). MOI treatment in SHR significantly reduced the plasma concentration of 8-isoprostane to a level similar to that observed in WKY (Figure 1(c)). These data are consistent with a vascular and systemic antioxidant effect of MOI treatment.

3.2. MOI Reduces NADPH Oxidase Expression and Upregulates SOD2. The NADPH oxidase (NOX) family of enzymes is a major source of ROS in the cardiovascular system. We thus next analyzed the effect of MOI on the aortic expression of four subunits of the active NOX2 enzyme complex. The expression of the catalytic subunit NOX2 (gp91 ${ }^{\text {phox }}$ ) and the expression of the regulatory subunits $\mathrm{p} 22^{\text {phox }}, \mathrm{p} 47^{\text {phox }}$, and $\mathrm{p} 67^{\text {phox }}$ tended to be higher in SHR than in WKY, although the differences did not reach significance (Figures 2(a), 2(b), and 2(c)). MOI treatment in SHR 


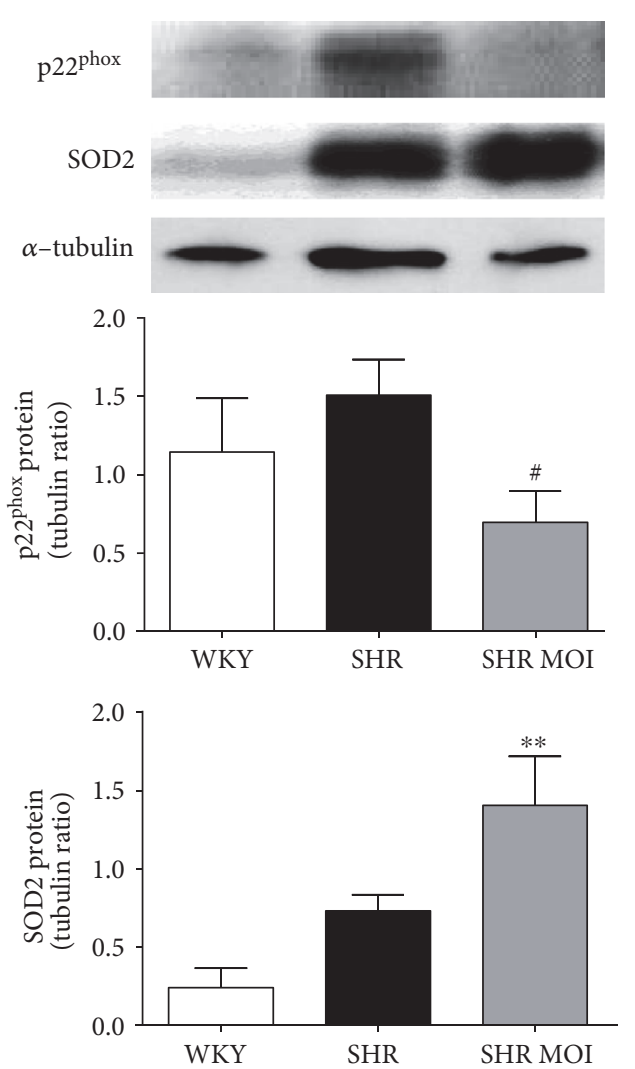

(a)
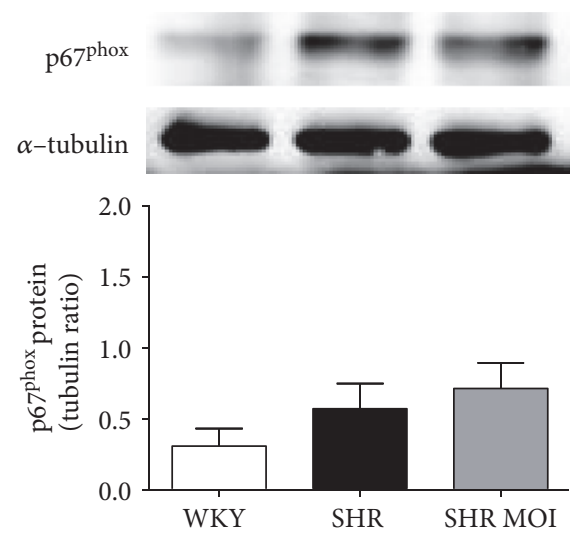

(c)
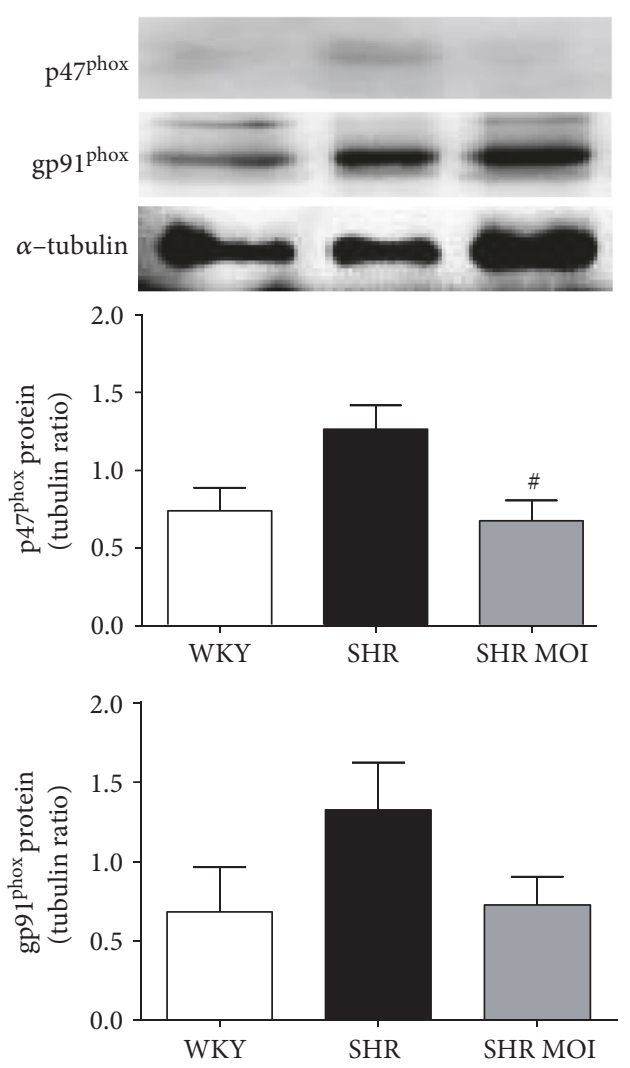

(b)

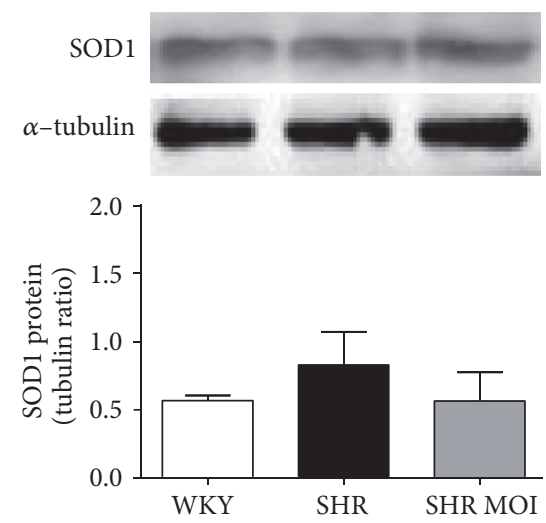

(d)

FIGURE 2: Representative Western blot and corresponding densitometric analysis of NADPH oxidase subunits (a-c) and SOD isoformes (a and d) in aortas from WKY rats (white plot), untreated SHR (SHR, black plot), and MOI-treated SHR (SHR MOI, grey plot). (a) p $22^{\text {phox }}\left(n=4,{ }^{*} p<0.05\right.$, SHR MOI versus SHR) and SOD2 protein expression $\left(n=4,{ }^{* *} p<0.01\right.$, SHR MOI versus WKY). (b) p47 ${ }^{\text {phox }}$ and gp $91^{\text {phox }}$ protein expression $\left(n=4-6,{ }^{\#} p<0.05\right.$, SHR MOI versus SHR or any significant difference, resp.). (c and d) p67 ${ }^{\text {phox }}$ and SOD1 protein expression, respectively $(n=4$, any significant difference). Data were normalized to $\alpha$-tubulin densitometry.

significantly decreased $\mathrm{p} 22^{\text {phox }}$ and $\mathrm{p} 47^{\text {phox }}$ expressions (Figures 2(a) and 2(b)). Regarding the antioxidant enzyme superoxide dismutase (SOD), SOD1 was expressed similarly in WKY, SHR, and SHR MOI (Figure 2(d)). The mitochondrial isoform of SOD, SOD2, equally expressed in WKY and SHR, was significantly increased in SHR MOI, suggesting that MOI treatment might improve arterial mitochondrial antioxidant activity (Figure 2(a)).
3.3. MOI Reduces Nitrotyrosine Expression in Rat Aortas. Oxidative stress is generally associated with nitrosative stress due to the overproduction of $\mathrm{NO}$ of inflammatory origin and its interaction with superoxide anion to form the reactive peroxynitrite anion $\left(\mathrm{ONOO}^{-}\right)$. Although there are multiple targets for the reactive $\mathrm{ONOO}^{-}$, the presence of 3-nitrotyrosine, whether free or protein bound, has been proposed as a marker of $\mathrm{ONOO}^{-}$formation. To assess the effect of MOI on nitrosative 

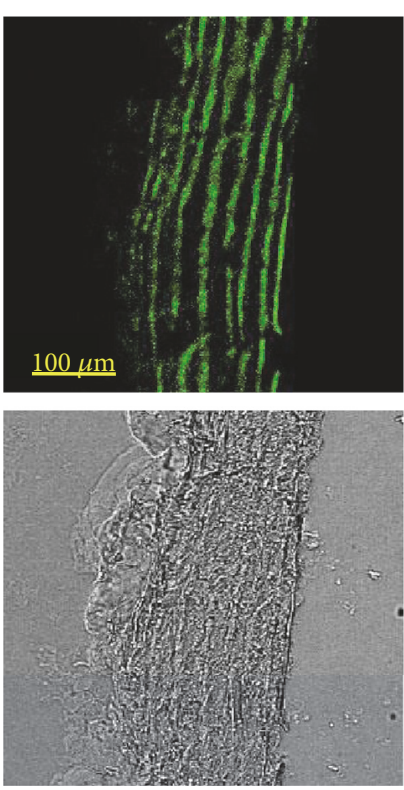

$\mathrm{C}(-)$
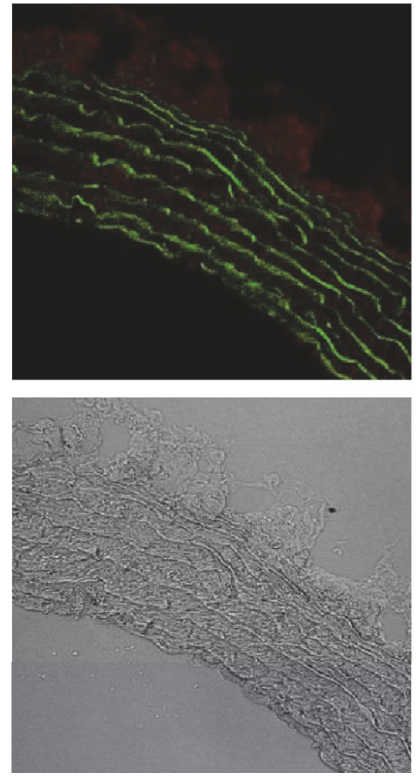

WKY
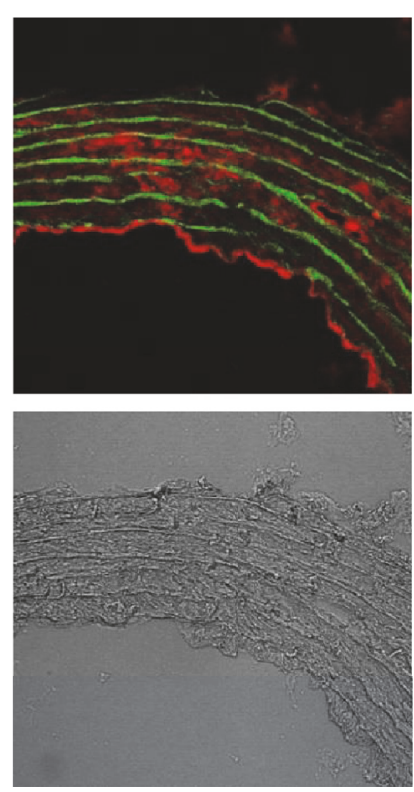

SHR
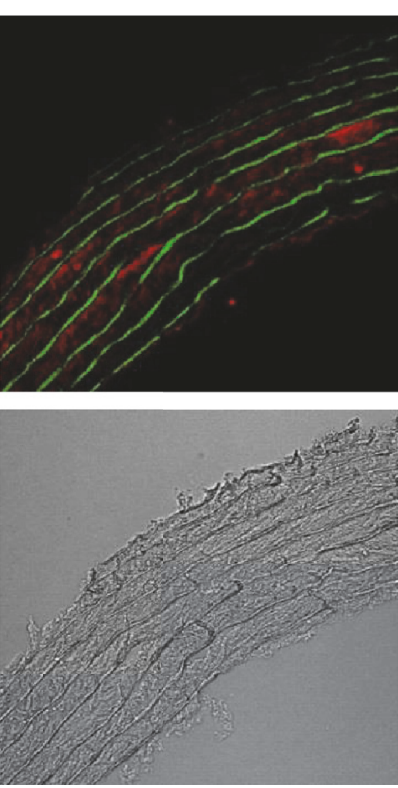

SHR MOI

(a)

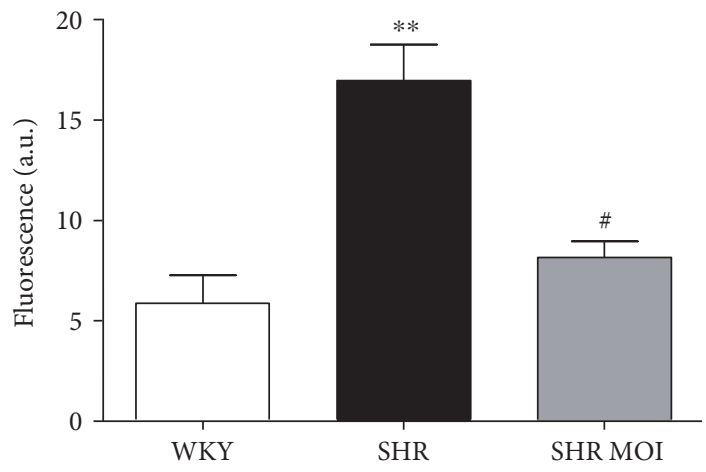

(b)

FIGURE 3: (a) Nitrosative stress (red staining), nitrotyrosine of proteins, in rat aortas from Wistar Kyoto (WKY), untreated SHR (SHR), and SHR treated with MOI (SHR MOI). Green fluorescence corresponds to autofluorescence of elastin. Below phase-contrast images. Negative control C(-), incubated only with the secondary murine fluorescent-labeled antibody, displays any specific red staining but only the green fluorescence of elastin. (b) Red fluorescence is expressed in arbitrary units (a.u.), $\left(n=4,{ }^{* *} p<0.01\right.$ SHR versus WKY and ${ }^{\#} p<0.05$ SHR MOI versus SHR).

stress, we thus performed immunostaining of nitrotyrosine in aorta sections. A strong staining was observed in both endothelium and the medial layer of SHR aortas compared to WKY (Figures 3(a) and 3(b)). In aortas from SHR MOI, nitrotyrosine staining was reduced to a level similar to that of WKY aortas (Figures 3(a) and 3(b)), indicating that MOI treatment decreased the enhanced nitrosative stress in hypertensive rats.

3.4. MOI Reduces iNOS Expression in Rat Aortas. To evaluate the anti-inflammatory properties of MOI treatment, we first analyze the expression of iNOS by immunostaining on aortic sections. No or weak red staining of iNOS was found in control WKY aortic sections while a marked staining was observed in both endothelium and the medial layer of SHR arteries, indicating upregulation of iNOS (Figures 4(a) and 4(b)). MOI treatment significantly reduced iNOS expression in SHR (Figures 4(a) and 4(b)). Assessment of circulating NO through measurement of plasma nitrite levels confirmed this observation, showing a strong increase of plasmatic nitrites in SHR group, which was significantly decreased in the SHR MOI group (Figure 4(c)).

3.5. MOI Reduces NFאB p65 Expression/Activation in Rat Aortas. The iNOS expression is induced, at least in part, through the activation of $\mathrm{NF} \kappa \mathrm{B}$ p65. We thus investigated the expression/activation of this transcription factor by immunostaining of p65 in aorta sections. The negative control obtained by incubation with the secondary murine fluorescent-labeled antibody did not display any red staining but only the green autofluorescence of the elastin (Figure 5(a)). A weak red staining of $\mathrm{NF} \kappa \mathrm{B}$ p65 was found in control WKY vessels while a marked staining was observed 

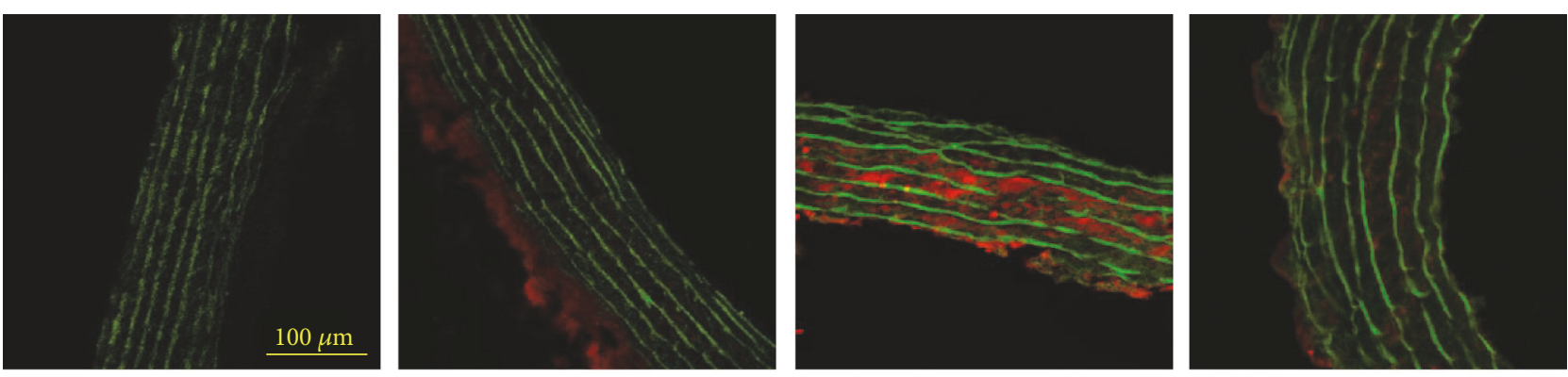

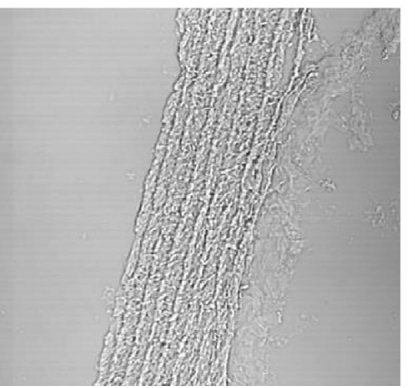

$\mathrm{C}(-)$

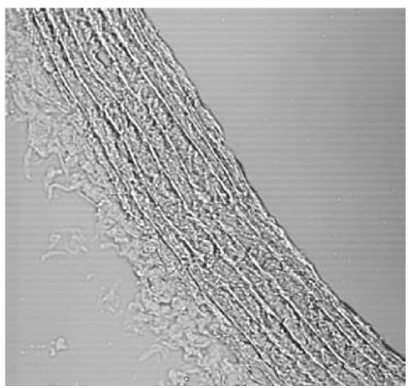

WKY

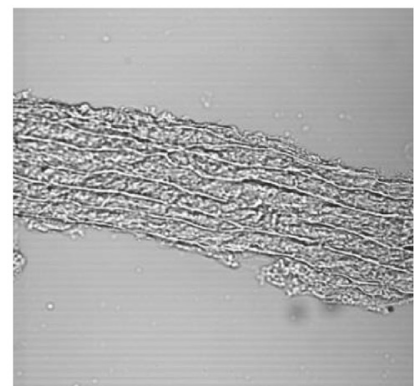

SHR

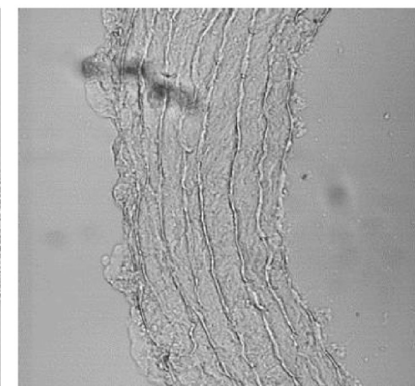

SHR MOI

(a)

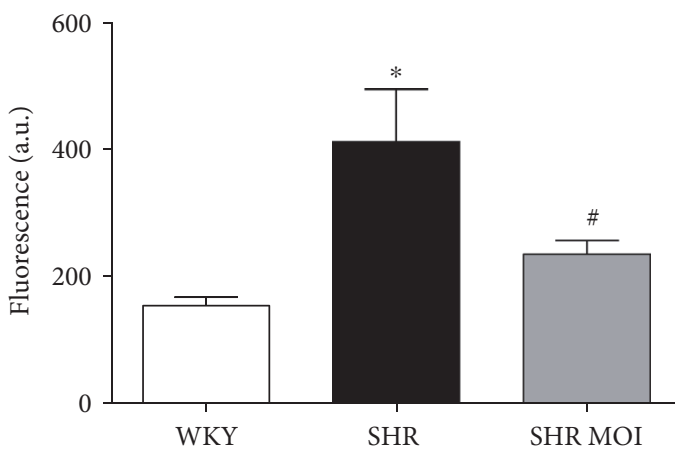

(b)

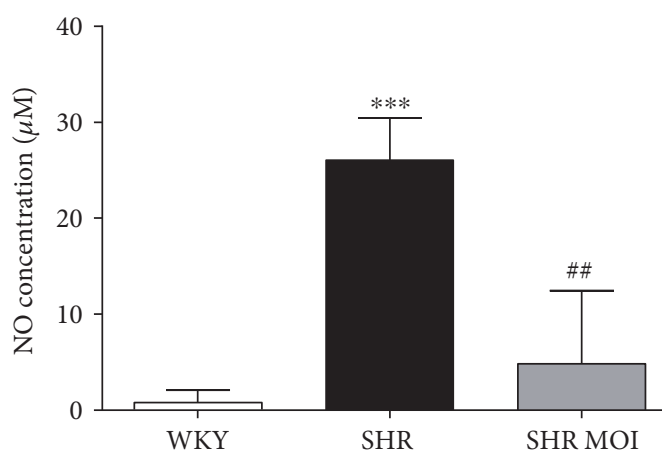

(c)

FIGURE 4: (a) Red staining of iNOS observed in rat aortas from Wistar Kyoto (WKY), untreated SHR (SHR), and SHR treated with MOI (SHR MOI). Green fluorescence corresponds to autofluorescence of elastin. Below phase-contrast images. Negative control C(-), incubated only with the secondary murine fluorescent-labeled antibody, displays any red staining but only the green fluorescence of elastin. (b) Red fluorescence is expressed in arbitrary units (a.u.), ( $n=4,{ }^{*} p<0.05$ SHR versus WKY and ${ }^{*} p<0.05$ SHR MOI versus SHR). (c) Plasmatic levels of nitrites expressed in $\mu \mathrm{M}\left(n=5-6,{ }^{* * *} p<0.001\right.$ SHR versus WKY and ${ }^{\# \#} p<0.01$ SHR MOI versus SHR).

in the vascular wall of SHR aortas (Figures 5(a) and 5(b)). MOI treatment significantly reduced the NFאB p65 red staining (Figures 5(a) and 5(b)). This beneficial anti-inflammatory effect of MOI seeds was confirmed by the measurement of plasmatic CRP (Figure 5(c)). CRP level, significantly increased in SHR, was reduced to a level similar to that of WKY by MOI (Figure 5(c)). These data confirm vascular and systemic anti-inflammatory properties of MOI seeds.

3.6. MOI Ameliorates Endothelial Function in Mesenteric Arteries. To test if MOI treatment was able to ameliorate vascular function in hypertensive rats, endothelial function was assessed in both aortic and mesenteric vessels. We found similar reduced endothelial-dependent relaxation to cumulative doses of carbachol in both SHR and SHR MOI aortas compared to WKY aortas, suggesting that MOI was not able to ameliorate endothelial function in conductance arteries (Figure 6(a)). However, MOI restored the altered carbachol-induced endothelial-dependent relaxation in resistance SHR mesenteric arteries (pEC50: 7.31 in WKY and 7.38 in SHR MOI versus 6.3 in SHR; Figure 6(b)).

\section{Discussion}

Our results provide evidence for beneficial vascular and systemic antioxidant and anti-inflammatory properties of MOI seeds in hypertension. Orally administrated, MOI seed powder limits the vascular oxidative and nitrosative stresses in aortas and improves the endothelial function of SHR resistance arteries.

High blood pressure is associated with increased reactive oxygen species (ROS) formation in vessels and target organs 

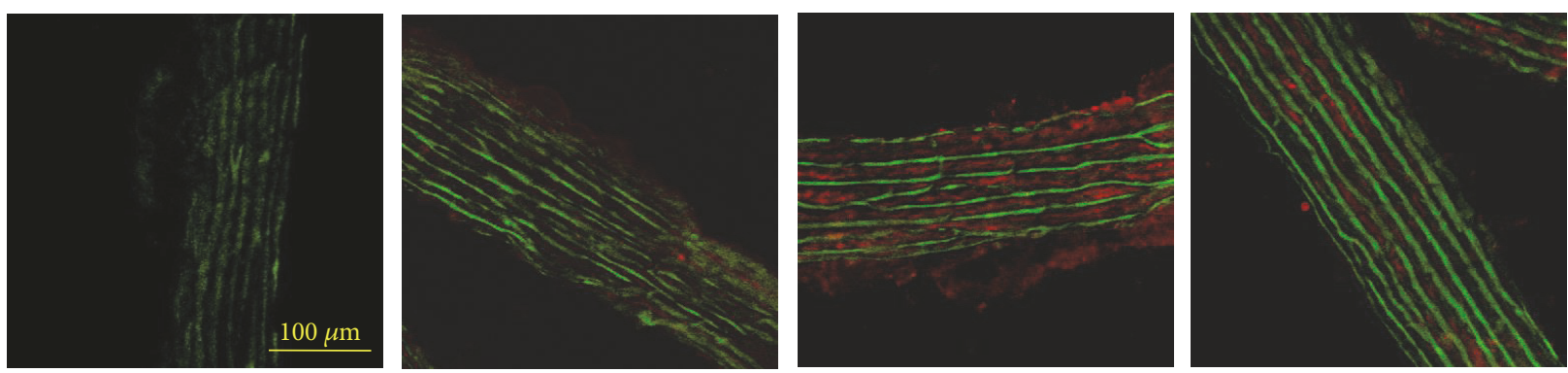

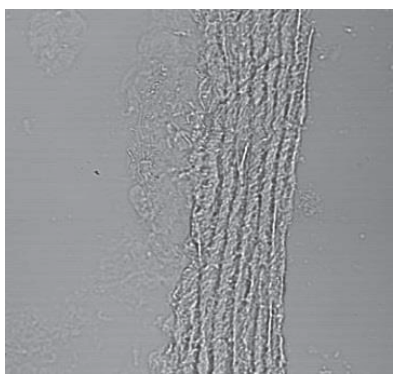

$\mathrm{C}(-)$

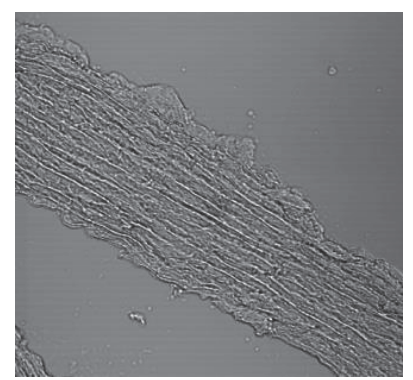

WKY

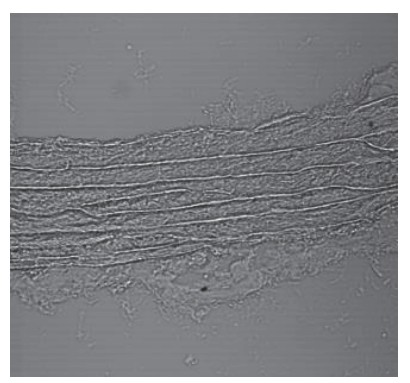

SHR

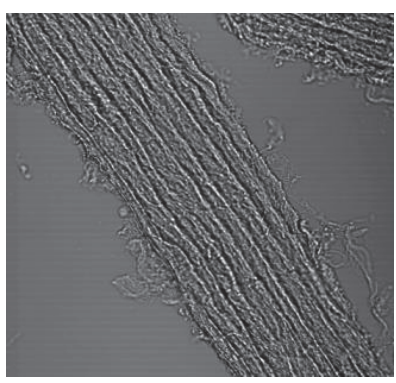

SHR MOI

(a)

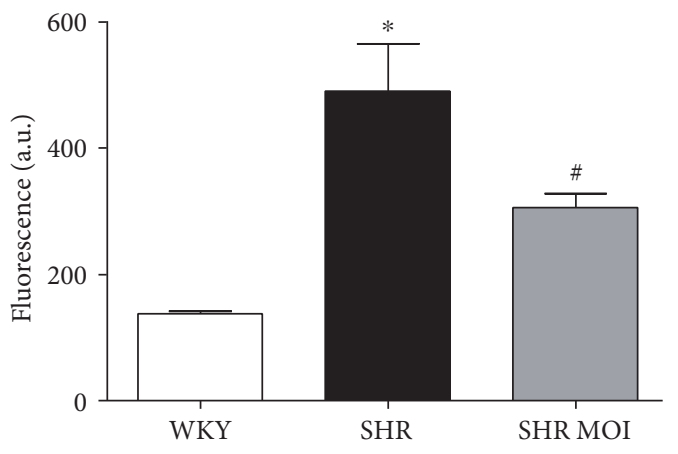

(b)

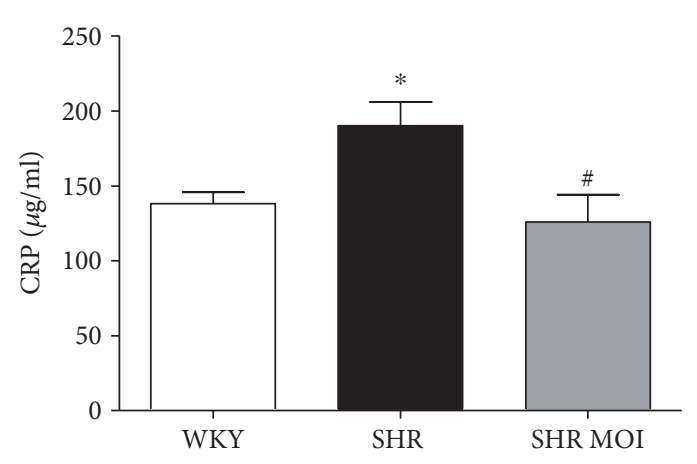

(c)

FIgURE 5: (a) Immunohistochemical red staining of the p65/RelA subunit of NF $\kappa$ B in rat aortas from Wistar Kyoto (WKY), SHR untreated (SHR), and SHR treated with MOI (SHR MOI). Green fluorescence corresponds to autofluorescence of elastin. Below phase-contrast images. Negative control $\mathrm{C}(-)$, incubated only with the secondary rabbit fluorescent-labeled antibody, displays any red staining but only the green fluorescence of elastin. (b) Red fluorescence is expressed in arbitrary units (a.u.) $\left(n=3-4,{ }^{*} p<0.05\right.$ SHR versus WKY and ${ }^{\#} p<0.05$ SHR MOI versus SHR). (c) Circulating level of C-reactive protein (CRP) $\left(n=5-7,{ }^{*} p<0.05\right.$ SHR versus WKY and ${ }^{\#} p<0.05$ SHR MOI versus SHR).

such as the brain and kidney [14]. ROS increase vascular dysfunction by promoting inflammation, thus establishing a positive feedback mechanism that participates to the development of hypertension [15]. $\mathrm{O}_{2}^{-}$, essentially of NOX origin, reduces endothelial NO bioavailability and promotes nitrosative stress leading to endothelial dysfunction and vascular tone increase that also contributes to hypertension [16-18]. SHR is a genetic model that exhibits many features of the human idiopathic hypertension. Indeed, hypertension in SHR follows the same progression as in humans, with prehypertensive, developing, and sustained hypertensive phases, each phase lasting at least several weeks $[19,20]$. Vascular oxidative stress precedes the increase in blood pressure and peripheral resistance in SHR [21,22], and administration of the antioxidant tempol prevents age-related development of hypertension in SHR, suggesting the therapeutic interest of targeting oxidative stress [23].
SHR is known to exhibit increased NOX-driven $\mathrm{O}_{2}^{-}$ generation in resistance and conducting arteries associated with NOX subunit overexpression and enhanced oxidase activity $[24,25]$. NOX-mediated $\mathrm{O}_{2}^{-}$production has also been observed in the vascular wall of patients with hypertension and atherosclerosis [26]. Here, we show that MOI reduced the expression of NOX regulatory subunits in SHR, suggesting that its antioxidant effect results, at least in part, from the downregulation of NOX subunits and the subsequent reduction of NOX-driven $\mathrm{O}_{2}^{-}$generation. We cannot exclude that MOI-induced decrease in NOXdependent $\mathrm{O}_{2}^{-}$generation might also indirectly result from the reduced level of circulating free 8-isoprostane in MOItreated SHR. 8-isoprostane is not only a marker of oxidative stress but it also increases, itself, oxidative stress by enhancing NOX expression and activity, thus creating a self-perpetuating positive feedback loop [27]. By leading 


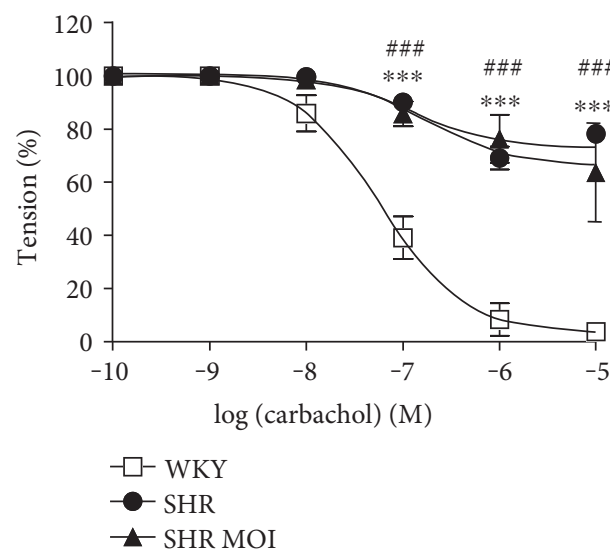

(a)

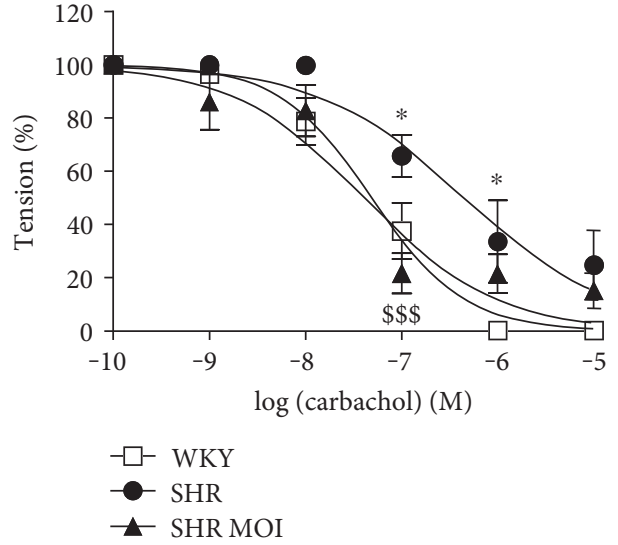

(b)

Figure 6: (a) Aorta and (b) mesenteric artery relaxation to cumulative doses of carbachol $\left({ }^{*} p<0.05\right.$ and ${ }^{* * *} p<0.001$ WKY versus SHR; $\# \# p<0.001 \mathrm{WKY}$ versus SHR MOI; ${ }^{\$ \$} p<0.001$ SHR versus SHR MOI, $n=5$ for each group of vessels, values are expressed as mean \pm S.E.M for each dose of carbachol).

to a reduction of this positive feedback mechanism, the MOI-induced reduction of free 8-isoprostane can thus participate to the decrease of $\mathrm{NOX}$-driven $\mathrm{O}_{2}^{-}$generation.

Furthermore, we found that MOI treatment in SHR also increases the aortic expression of SOD2, a mitochondrial Mn-containing enzyme described as one of the most important protective systems against oxidative stress [28]. SOD2 downregulation is proportionally linked to increased cell apoptosis under stress conditions while SOD2 upregulation reduces cell death and ROS levels during oxidative stress [29]. SOD2 upregulation is associated with the improvement of endothelial function in experimental models of diabetes, hypertension, and metabolic syndrome $[30,31]$. Thus, the antioxidant action of MOI is likely due to both a reduction of $\mathrm{O}_{2}^{-}$production by the downregulation of $\mathrm{p} 22^{\text {phox }}$ and $\mathrm{p} 47^{\text {phox }}$ and the increased $\mathrm{O}_{2}^{-}$elimination through SOD2 upregulation. Such a stimulation of antioxidant mechanism has been previously described for another Malagasy plant, Cedrelopsis grevei, used as an antiherpertensive treatment. This plant upregulated another SOD, the $\mathrm{Cu} / \mathrm{Zn} \mathrm{SOD}$, and improved endothelial function in rats [8].

Oxidative stress is mainly associated with inflammation and NO overproduction by iNOS upregulation through the activity of transcription factor $\mathrm{NF} \kappa \mathrm{B}$ p65 [32]. NO reacts with $\mathrm{O}_{2}^{-}$, leading to peroxynitrite production, subsequent protein tyrosine nitration, and cell toxicity [17]. In agreement with the antioxidant effect of MOI, we observed a reduction of nitrosative stress in aortas from SHR MOI associated with a reduction of $\mathrm{NF} \kappa \mathrm{B}$ p65 expression/activation and iNOS expression, thus revealing an anti-inflammatory action of MOI. A systemic anti-inflammatory effect of MOI treatment was confirmed by the low circulating levels of CRP in SHR MOI compared to SHR.

Functionally, we found that MOI treatment improves endothelial relaxation in resistance arteries in SHR. Oxidative stress is known to be causally involved in the endothelial dysfunction associated with hypertension [16-18]. Our results thus further support the beneficial effect of antioxidants on endothelial function in hypertensive condition and the therapeutic interest of plants such as MOI that target oxidative stress.

The complete composition of MOI seeds has been previously described [33, 34]. The beneficial vascular effects observed in the present work are probably due to the presence in the seeds of tocopherols, phenolic acids (gallic and ferulic acids), and flavonoids (such as quercetin, catechin, and epicathechin) known for their potential antioxidant activity [34]. Moreover, we previously identified glucosinolates, in particular glucomoringin, in MOI seeds extract that can also contribute to its cardiovascular protective role [11]. Glucomoringin hydrolysis by myrosinase activity during food processing or digestion leads to the formation of isothiocianates (ITCs) [35]. Myrosinase is an enzyme acting during vegetal cell lysis and death [36]. It was previously demonstrated that the ITCs produced from glucosinolates of Brassicaceae inhibited NF- $\kappa \mathrm{B}$ and prevented oxidative stress in several oxidative and inflammatory diseases $[37,38]$. Furthermore, glucoeurocin-derived ITCs contained in rocket were shown to have indirect antioxidant activity by inducing phase-II enzymes that play an important role in the detoxification of electrophiles and subsequent free radical level reduction [39]. More recently, the anti-inflammatory and antioxidant activity of the glucomoringin-derived ITC moringin has been described in LPS-stimulated macrophages when associated with cannabidiol [40]. In agreement with these previous findings, glucomoringin ITCs could thus participate, in association with other compounds such as tocopherols and flavonoids, in the in vivo antioxidant and anti-inflammatory cardiovascular effects evidenced in MOI SHR. The beneficial effects of whole MOI seeds are thus likely due to their original composition and the synergistic action of several antioxidant compounds rather to a unique active compound.

\section{Conclusions}

In conclusion, the present study demonstrates the antioxidant and anti-inflammatory effect of orally administrated 
MOI seeds and their beneficial action on the endothelial function in a rat model of hypertension. The present study thus supports scientifically the empirical use of MOI in traditional Malagasy medicine to treat high blood pressure and other cardiovascular disorders associated with inflammation and oxidative stress.

$\begin{array}{ll}\text { Abbreviations } \\ \text { CRP: } & \text { C-reactive protein } \\ \text { DHE: } & \text { Dihydroethidine } \\ \text { iNOS: } & \text { Inducible NO synthase } \\ \text { ITCs: } & \text { Isothiocyanates } \\ \text { MOI: } & \text { Moringa oleifera } \\ \text { NO: } & \text { Nitric oxide } \\ \mathrm{NO}_{2}^{-}: & \text {Nitrite } \\ \mathrm{O}_{2}^{-}: & \text {Superoxide anion } \\ \mathrm{ONOO}^{-}: & \text {Peroxynitrite } \\ \text { SHR: } & \text { Spontaneously hypertensive rats } \\ \text { SHR MOI: } & \text { SHR treated with Moringa oleifera seeds } \\ \text { SOD: } & \text { Superoxide dismutase } \\ \text { WKY: } & \text { Wistar Kyoto rats. }\end{array}$

\section{Conflicts of Interest}

The authors declare that there is no conflict of interest regarding the publication of this article.

\section{Authors' Contributions}

Gervaise Loirand and Angela Tesse contributed equally to this work.

\section{Acknowledgments}

We thank Philippe Hulin and Steven Nedellec and the platform MicroPicell (SFR Francois Bonamy, Nantes) for the technical assistance in confocal microscopy and image analysis. We thank Séverine Derbré and Serge Michalet for the interesting scientific discussion about the composition of MOI seeds. We also value the support provided by the animal facility unit of the University of Nantes. This work is supported by grants from INSERM and Région Pays de la Loire (PROVASC project).

\section{References}

[1] A. V. Chobanian, G. L. Bakris, H. R. Black et al., "Seventh report of the joint National committee on prevention, detection, evaluation, and treatment of high blood pressure," Hypertension, vol. 42, pp. 1206-1252, 2003.

[2] R. Lozano, M. Naghavi, K. Foreman et al., "Global and regional mortality from 235 causes of death for 20 age groups in 1990 and 2010: a systematic analysis for the global burden of disease study 2010," Lancet, vol. 380, no. 9859, pp. 2095-2128, 2012.

[3] S. S. Lim, T. Vos, A. D. Flaxman et al., "A comparative risk assessment of burden of disease and injury attributable to 67 risk factors and risk factor clusters in 21 regions, 1990-2010: a systematic analysis for the Global Burden of Disease Study 2010," Lancet, vol. 380, no. 9859, pp. 2224-2260, 2012.

[4] WHO, Global status report on noncommunicable diseases, Geneva, World Health Organization, 2014, http://apps.who. int/iris/bitstream/10665/148114/1/9789241564854_eng.pdf?ua=1.

[5] M. Di Cesare, J. E. Bennett, N. Best, G. A. Stevens, G. Danaei, and M. Ezzati, "The contributions of risk factor trends to cardiometabolic mortality decline in 26 industrialized countries," International Journal of Epidemiology, vol. 42, no. 3, pp. 838-848, 2013.

[6] J. W. Novy, "Medicinal plants of the eastern region of Madagascar," Journal of Ethnopharmacology, vol. 55, pp. 119-126, 1997.

[7] E. F. Rakotoniriana, J. F. Rajaonarison, E. G. Raoelison et al., "Animicrobial activity of 23 endemic plants in Madagascar," Tropical Journal of Pharmaceutical Research, vol. 9, pp. 165$171,2010$.

[8] H. Ralay-Ranaivo, O. Rakotoarison, A. Tesse et al., "Cedrelopsis grevei induced hypotension and improved endothelial vasodilatation through an increase of $\mathrm{Cu} / \mathrm{Zn}$-SOD protein expression," American Journal of Physiology. Heart and Circulatory Physiology, vol. 286, pp. H775-H781, 2004.

[9] G. Rakotomalala, C. Agard, P. Tonnerre et al., "Extract from Mimosa pigra attenuates chronic experimental pulmonary hypertension," Journal of Ethnopharmacology, vol. 148, no. 1, pp. 106-116, 2013.

[10] G. C. Stevens, K. P. Baiyeri, and O. Akkinnnagbe, "Ethnomedicinal and culinary uses of Moringa oleifera Lam. In Nigeria," Journal of Medicinal Plants Research, vol. 7, no. 13, pp. 799-804, 2013.

[11] J. I. Randriamboavonjy, G. Loirand, N. Vaillant et al., "Cardiac protective effects of Moringa oleifera seeds in spontaneously hypertensive rats," American Journal of Hypertension, vol. 29, no. 7, pp. 873-881, 2016.

[12] H. A. Ogbunugafor, F. U. Eneh, A. N. Ozumba et al., "Physicochemical and antioxidant properties in Moringa oleifera seed oil," Pakistan Journal of Nutrition, vol. 10, no. 5, pp. 409414, 2011.

[13] F. J. Miller, D. D. Gutterman, C. D. Rios, D. D. Heistad, and B. L. Davidson, "Superoxide production in vascular smooth muscle contributes to oxidative stress and impaired relaxation in atherosclerosis," Circulation Research, vol. 82, pp. 12981305, 1998.

[14] T. Fukai and M. Ushio-Fukai, "Superoxide dismutases: role in redox signaling, vascular function, and diseases," Antioxidants \& Redox Signaling, vol. 15, pp. 1583-1606, 2011.

[15] S. Xu and R. M. Touyz, "Reactive oxygen species and vascular remodelling in hypertension: still alive," The Canadian Journal of Cardiology, vol. 22, pp. 947-951, 2006.

[16] M. McIntyre, D. F. Bohr, and A. F. Dominiczak, "Endothelial function in hypertension: the role of superoxide anion," Hypertension, vol. 34, pp. 539-545, 1999.

[17] S. W. Ballinger, C. Patterson, C. N. Yan et al., "Hydrogen peroxide-and peroxynitrite-induced mithocondrial DNA damage and dysfunction in vascular endothelial and smooth muscle cells," Circulation Research, vol. 86, pp. 960-966, 2000.

[18] L. E. Spieker, G. Noll, F. T. Ruschitzka, W. Maier, and T. F. Luscher, "Working under pressure: the vascular endothelium in arterial hypertension," Journal of Human Hypertension, vol. 14, pp. 617-630, 2000. 
[19] S. A. Doggrell and L. Brown, "Rat models of hypertension, cardiac hypertrophy and failure," Cardiovascular Research, vol. 39, no. 1, pp. 89-105, 1998.

[20] S. Amoureux, L. Lorgis, P. Sicard, C. Girard, L. Rochette, and C. Vergely, "Vascular BDNF expression and oxidative stress during aging and the development of chronic hypertension," Fundamental \& Clinical Pharmacology, vol. 26, no. 2, pp. 227-234, 2012.

[21] S. Grinfeld, C. A. Hamilton, S. Mesaros et al., "Role of superoxide in the depressed nitric oxide production by the endothelium of genetically hypertensive rats," Hypertension, vol. 26, pp. 854-857, 1995.

[22] S. Kerr, M. J. Brosnan, M. McIntyre, J. L. Reid, A. F. Dominiczak, and C. A. Hamilton, "Superoxide anion production is increased in a model of genetic hypertension role of the endothelium," Hypertension, vol. 33, pp. 1353-1358, 1999.

[23] L. Nabha, J. C. Garbern, C. L. Buller, and J. R. Charpie, "Vascular oxidative stress precedes high blood pressure in spontaneously hypertensive rats," Clinical and Experimental Hypertension, vol. 27, pp. 71-82, 2005.

[24] G. Zalba, F. J. Beaumont, G. San José et al., "Vascular $\mathrm{NADH} / \mathrm{NADPH}$ oxidase is involved in enhanced superoxide production in spontaneously hypertensive rats," Hypertension, vol. 35, no. 5, pp. 1055-1061, 2000.

[25] R. M. Touyz, "Reactive oxygen species, vascular oxidative stress, and redox signaling in hypertension: what is the clinical significance?" Hypertension, vol. 44, no. 3, pp. 248-252, 2004.

[26] M. U. Moreno, G. San José, J. Orbe et al., "Preliminary characterisation of the promoter of the human $\mathrm{p} 22^{\text {phox }}$ gene: identification of a new polymorphism associated with hypertension," FEBS Letters, vol. 542, no. 1-3, pp. 27-31, 2003.

[27] S. Muzaffar, N. Shukla, C. Lobo, G. D. Angelini, and J. Y. Jeremy, "Iloprost inhibits superoxide formation and gp91 ${ }^{\text {phox }}$ expression induced by the thromboxane $A_{2}$ analogue U46619, 8-isoprostane $\mathrm{F}_{2 \alpha}$, prostaglandin $\mathrm{F}_{2 \alpha}$, cytokines and endotoxin in the pig pulmonary artery," British Journal of Pharmacology, vol. 141, no. 3, pp. 488-496, 2004.

[28] I. Fridovich and B. Freeman, "Antioxidant defenses in the lung," Annual Review of Physiology, vol. 48, pp. 693-702, 1986.

[29] H. Van Remmen, M. D. Williams, Z. Guo et al., "Knockout mice heterozygous for Sod2 show alterations in cardiac mitochondrial function and apoptosis," The American Journal of Physiology-Heart and Circulatory Physiology, vol. 281, pp. H1422-H1432, 2001.

[30] F. M. Faraci and S. P. Didion, "Vascular protection: superoxide dismutase isoforms in the vessel wall," Arteriosclerosis, Thrombosis, and Vascular Biology, vol. 24, pp. 1367-1373, 2004.

[31] D. D. Heistad, "Oxidative stress and vascular disease: 2005 duff lecture," Arteriosclerosis, Thrombosis, and Vascular Biology, vol. 26, pp. 689-695, 2006.

[32] P. P. Tak and G. S. Firestein, "NF-kappaB: a key role in inflammatory diseases," The Journal of Clinical Investigation, vol. 107, pp. 7-11, 2001.

[33] M. Premi and H. K. Sharma, "Effect of extraction conditions on the bioactive compounds from Moringa oleifera (PKM 1) seeds and their identification using LC-MS," Journal of Food Measurement and Characterization, vol. 11, pp. 213-225, 2017.

[34] A. Leone, A. Spada, A. Battezzati, A. Schiraldi, J. Aristil, and S. Bertoli, "Moringa oleifera Seeds and oil: characteristics and uses for human health," International Journal of Molecular Sciences, vol. 17, no. 12, 2016.
[35] I. T. Johnson, "Phytochemicals and cancer," The Proceedings of the Nutrition Society, vol. 66, pp. 207-215, 2007.

[36] I. Hara-Nishimura and N. Hatsugai, "The role of vacuole in plant cell death," Cell Death and Differentiation, vol. 18, no. 8, pp. 1298-1304, 2011.

[37] D. Brunelli, M. Tavecchio, C. Falcioni et al., "The isothiocyanate produced from glucomoringin inhibits NF-kB and reduces myeloma growth in nude mice in vivo," Biochemical Pharmacology, vol. 79, no. 8, pp. 1141-1148, 2010.

[38] G. Sita, P. Hrelia, A. Tarozzi, and F. Morroni, "Isothiocyanates are promising compounds against oxidative stress, neuroinflammation and cell death that may benefit neurodegeneration in Parkinson's disease," International Journal of Molecular Sciences, vol. 17, no. 9, 2016.

[39] J. Barillari, D. Canistro, M. Paolini et al., "Direct antioxidant activity of purified glucoerucin, the dietary secondary metabolite contained in rocket (Eruca sativa mill.) seeds and sprouts," Journal of Agricultural and Food Chemistry, vol. 53, pp. 24752482, 2005.

[40] T. S. Rajan, S. Giacoppo, R. Iori et al., "Anti-inflammatory and antioxidant effects of a combination of cannabidiol and moringin in LPS-stimulated macrophages," Fitoterapia, vol. 112, pp. 104-115, 2016. 


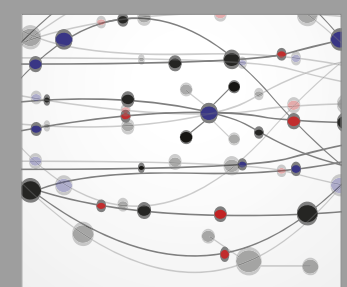

The Scientific World Journal
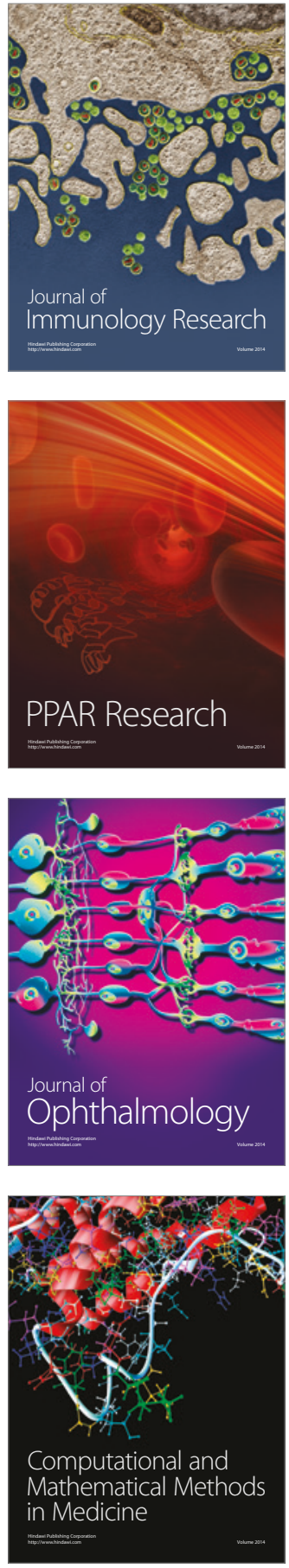

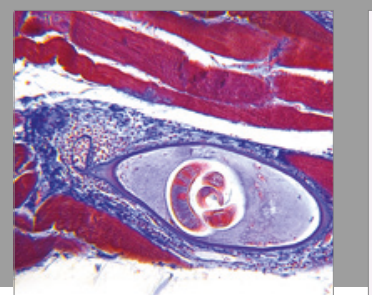

Gastroenterology Research and Practice
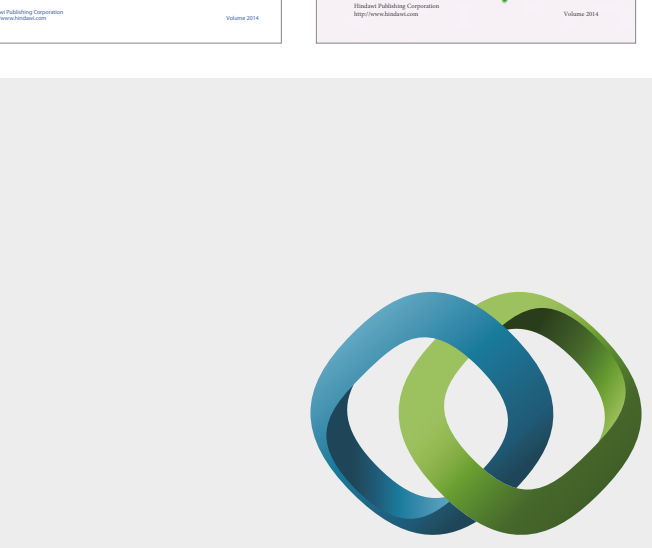

\section{Hindawi}

Submit your manuscripts at

https://www.hindawi.com
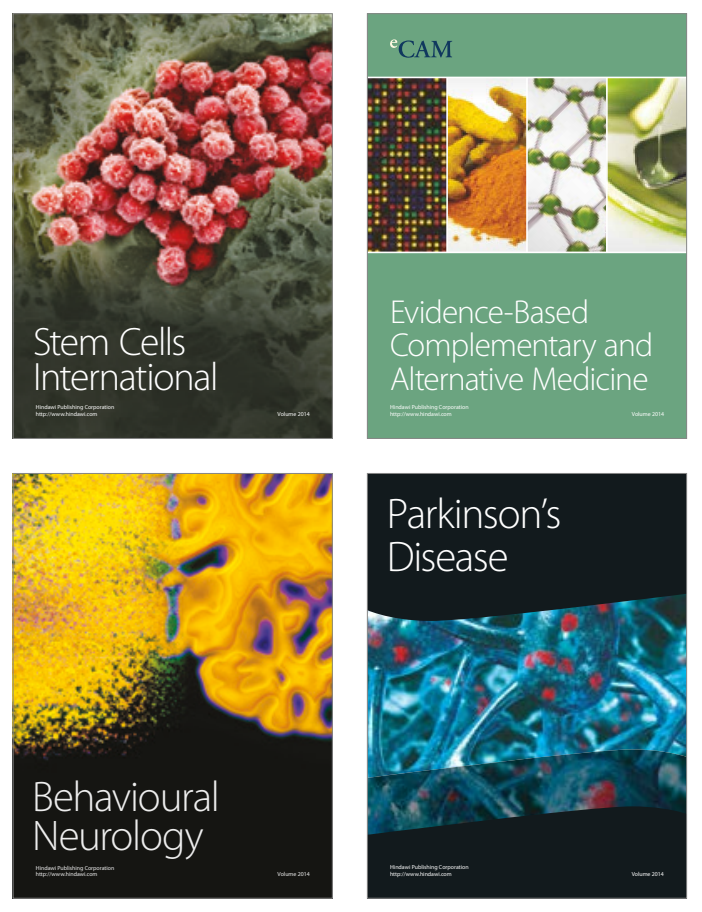
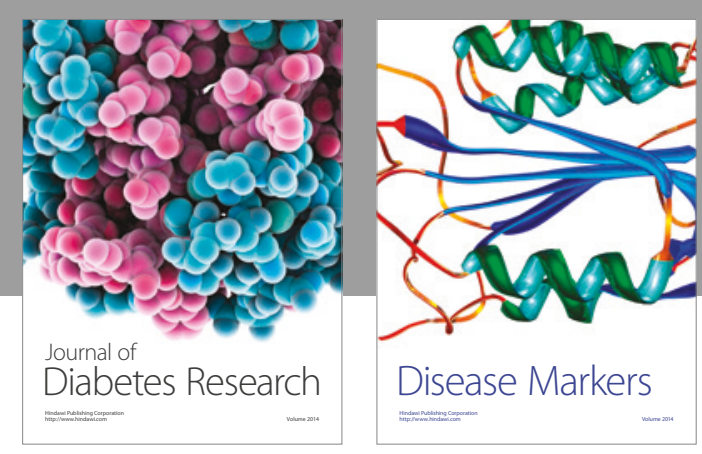

Disease Markers
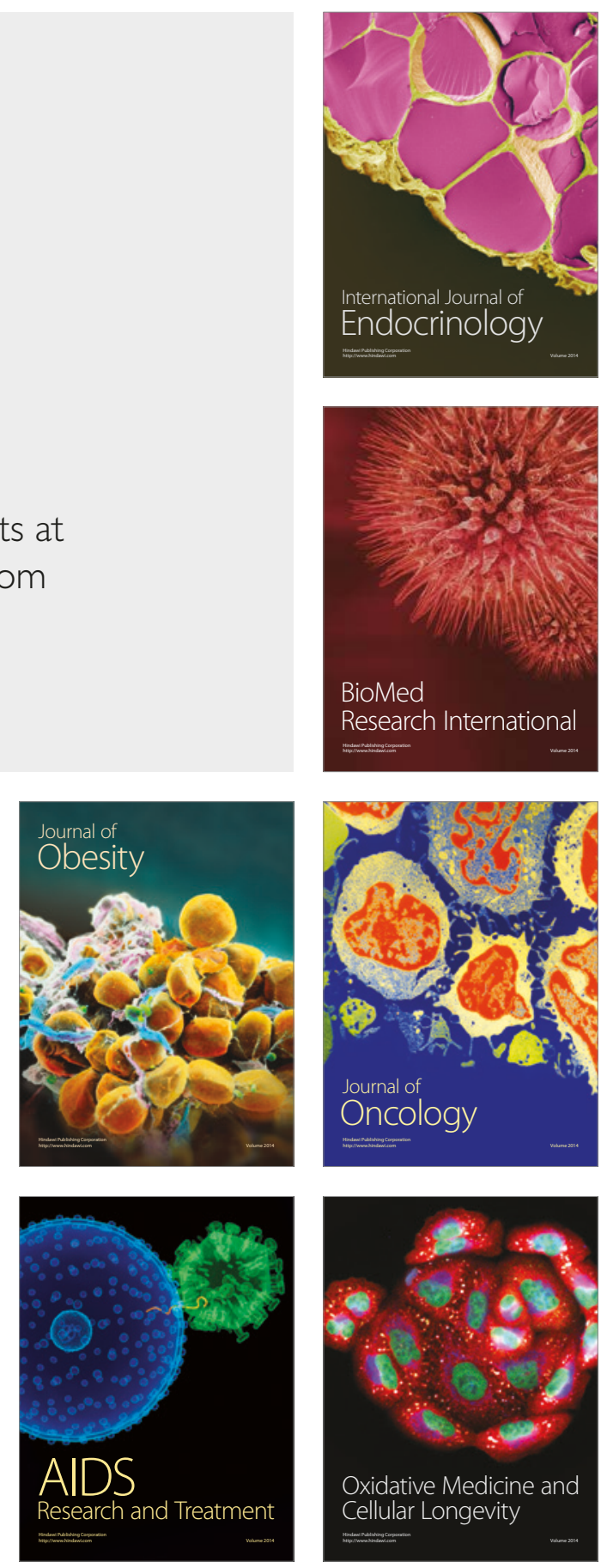